\title{
Corrigendum: Duplication of a promiscuous transcription factor drives the emergence of a new regulatory network
}

Ksenia Pougach, Arnout Voet, Fyodor A. Kondrashov, Karin Voordeckers, Joaquin F. Christiaens, Bianka Baying, Vladimir Benes, Ryo Sakai, Jan Aerts, Bo Zhu, Patrick Van Dijck \& Kevin J. Verstrepen

Nature Communications 5:4868 doi: 10.1038/ncomms5868 (2014); Published 10 Sep 2014; Updated 26 Feb 2015

Two affiliations for Fyodor A. Kondrashov were omitted from this Article. The correct affiliations are given below:

Bioinformatics and Genomics Programme, Centre for Genomic Regulation (CRG) 88 Dr. Aiguader, 08003 Barcelona, Spain.

Universitat Pompeu Fabra (UPF), 08003 Barcelona, Spain.

Institució Catalana de Recerca i Estudis Avançats (ICREA), 23 Pg. Lluís Companys, 08010 Barcelona, Spain. 